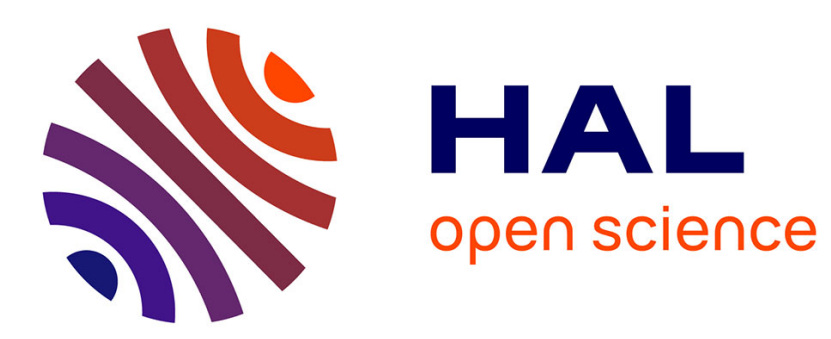

\title{
Propagation de la combustion sur un réseau hétérogène bidimensionnel
}

Jean Nahmias, Hervé Téphany, E. Guyon

\section{To cite this version:}

Jean Nahmias, Hervé Téphany, E. Guyon. Propagation de la combustion sur un réseau hétérogène bidimensionnel. Revue de Physique Appliquée, 1989, 24 (7), pp.773-777. 10.1051/rphysap:01989002407077300 . jpa-00246100

\section{HAL Id: jpa-00246100 https://hal.science/jpa-00246100}

Submitted on 1 Jan 1989

HAL is a multi-disciplinary open access archive for the deposit and dissemination of scientific research documents, whether they are published or not. The documents may come from teaching and research institutions in France or abroad, or from public or private research centers.
L'archive ouverte pluridisciplinaire HAL, est destinée au dépôt et à la diffusion de documents scientifiques de niveau recherche, publiés ou non, émanant des établissements d'enseignement et de recherche français ou étrangers, des laboratoires publics ou privés. 
Classification

Physics Abstracts

$44.00-05.50$

\title{
Propagation de la combustion sur un réseau hétérogène bidimensionnel
}

\author{
Jean Nahmias ( $\left.{ }^{1}\right)$, Hervé Téphany $\left(^{2}\right)$ et E. Guyon $\left({ }^{3}\right)$ \\ (1) Consultant en mécanique des fluides, Chargé d'enseignement à l'Université Paris Sud, 14 rue du St. \\ Gothard, 75014 Paris, France \\ (2) 36 rue Carnot, 92370 Chaville, France \\ (3) E.S.P.C.I., U.A. 857 du CNRS, 10 rue Vauquelin, 75231 Paris Cedex 05, France
}

(Reçu le 5 janvier 1989, accepté le 29 mars 1989)

\begin{abstract}
Résumé. - Nous avons construit un modèle sur réseau carré, comportant des sites combustibles et non combustibles aléatoirement distribués en pourcentages variables ( $q$ étant la fraction des éléments non combustibles), afin d'étudier l'influence de cette distribution sur la propagation du feu. En absence de vent, la propagation est compatible avec un modèle de percolation d'invasion sur un réseau carré de sites avec interaction de premier plus proche voisin conduisant à un seuil peu différent de la valeur théorique $q_{\mathrm{c}}=0,41$. Le seuil est plus grand quand le vent souffle sur le modele. La valeur maximale du seuil obtenue est $q_{\mathrm{c}}=0,65$. L'observation de l'état final du modèle permet de dégager les caractères dirigé, non local et corrélé de la contagion.
\end{abstract}

\begin{abstract}
We have built a square network model containing combustible and non combustible blocks randomly distributed, with a variable concentration ( $q$ is the fraction of non combustible elements) in order to study the effect of randomness on the propagation of fire. In the absence of wind, the propagation is consistent with a model of invasion percolation on a square site lattice with nearest neighbour interaction leading to a threshold not far from the theoretical value $q_{\mathrm{c}} \cong 0.39$. The threshold is larger when wind blows on model. The largest threshold value obtained was $q_{\mathrm{c}}=0.65$. The observation of the final state of the model permits to bring out the directed, non-local and correlated characters of the contagion.
\end{abstract}

Divers modèles numériques de simulation de la propagation des feux ont été développés en relation avec la prévention des incendies [1]. Dans le cas des incendies de forêt, ces modèles utilisent un maillage discret de la zone combustible en attribuant à chaque maille des paramètres liés à la topographie locale, les qualités de combustion de la végétation en plus de paramètres plus globaux tels que le vent ou l'humidité. Les calculs décrivent alors une contagion du feu de proche en proche et, convenablement ajustés, conduisent à une dynamique de front en bon accord avec les données réelles en particulier dans le cas de zones forestières étendues et assez homogènes. Des expériences modèles utilisant des lits réguliers de particules ont été aussi conduites dans des tunnels aérodynamiques [2] et conduisent généralement à des profils réguliers de front. A l'opposé, dans le cas de zones où la végétation et les conditions géographiques sont très hétérogènes, l'utilisation d'une approche du type percolation, peut sembler intéressante [3]. De fait, différents modèles faisant explicitement référence à la propagation des incendies ont été formulés. Une première approche [4] avait utilisé un réseau carré de liens dans lequel les arbres, supposés isolés les uns des autres comme dans un verger, occupent les nœuds du réseau. La probabilité pour que le feu «saute » d'un arbre à un autre est une fonction rapidement décroissante de la distance des arbres entre eux, ainsi que de variables, caractéristiques de la nature des espèces végétales et des conditions extérieures locales. Un tel mécanisme de transfert peut être rapproché du transport par saut activé des électrons dans les semiconducteurs désordonnés [5]. Il est de plus possible d'introduire dans ce modèle des effets de corrélation entre sites [6]. Récemment plusieurs modèles basés sur les notions de percolation [7] ont été développés. Citons les travaux de Grassberger [8], repris par McKay et 
Jan [9], ainsi que ceux de Albinet et al. [10]. Ces modèles permettent de prendre en compte la dynamique de la propagation du feu, en introduisant des durées de combustion des arbres et des durées d'échauffement des arbres voisins. Dans [9], il s'agit d'un modèle de percolation de liens et dans [10] d'une percolation de sites. Dans ce dernier cas, on a envisagé des interactions de sites possibles au-delà du premier plus proche voisin. Sur le même type de modèle une tentative d'introduction de l'influence du vent a été effectuée [11].

A notre connaissance, cependant, ces modèles n'ont pas fait l'objet de comparaison avec des situations réelles ou modèles. Dans cette note nous présentons une simulation expérimentale directe de la propagation d'un feu, en absence ou en présence de vent, dégageant des caractéristiques de la percolation et suggérant la nécessité de modèles plus élaborés.

Le modèle physique utilisé se divise en trois zones (Fig. 1). La première (zone 1) est uniquement constituée de combustible. Elle est suivie d'une deuxième zone dans laquelle sont distribués aléatoirement des éléments combustibles et non combustibles de forme carrée. Une troisième zone entièrement combustible, située après la zone 2 , permet de détecter, si elle s'enflamme, le passage du feu à travers la zone 2 . Les éléments combustibles et non combustibles sont des pavés carrés de dimensions de $15 \mathrm{~mm} \times 15 \mathrm{~mm}$ et $3 \mathrm{~mm}$ d'épaisseur. Les zones 1,2 et 3 ont pour longueur $525 \mathrm{~mm}$ et largeurs respectives $75 \mathrm{~mm}$, $300 \mathrm{~mm}, 30 \mathrm{~mm}$ (c'est-à-dire 35 éléments selon la longueur et respectivement 5,20 et 2 éléments selon la largeur). La zone 2 comporte donc 700 sites, nombre acceptable eu égard aux effets de taille des réseaux, qui doivent être pris en compte dans l'application de la théorie. Le combustible, à base de sciure de bois et de paraffine fortement compactées, a été débité dans des petits blocs. Le non combustible est taillé dans des plaques de fibrociment de qualité courante. L'épaisseur de $3 \mathrm{~mm}$ a été retenue pour réaliser un compromis entre la facilité de fabrication des petits carrés, le caractère $2 \mathrm{D}$ du modèle et la hauteur des flammes, car celle-ci croît rapidement avec l'épaisseur du combustible. Le rapport hauteur de flamme/épaisseur du combustible a une valeur de l'ordre de 30 pour le foyer de la zone 1 pleinement développé.

Deux séries d'expériences ont été effectuées, sans et avec vent. Lors de ces expériences la mise à feu s'effectue simultanément sur toute la première ligne de la zone 1. Sans vent nous laissons le feu se propager sur le réseau jusqu'à l'extinction. Les pourcentages indiqués par la suite pour caractériser la distribution des sites dans la zone 2 sont définis comme le rapport $q$ du nombre de sites non combustibles au nombre de sites total.

1) Sans vent, la « contamination » des sites se fait uniquement par les premiers plus proches voisins. Les expériences montrent que, pour des pourcentages d'éléments non combustibles $q(0 \leqq q \leqq 1)$ strictement supérieurs à 0,45 , le feu ne traverse pas la zone 2. Les flammes progressent à partir de la zone 1 dans les amas de combustible qui leur sont offerts et s'arrêtent en l'absence d'amas combustible «infini " (en percolation on utilise abusivement l'expression amas « infini » pour indiquer l'amas qui relie les faces opposées d'un réseau de taille finie au-

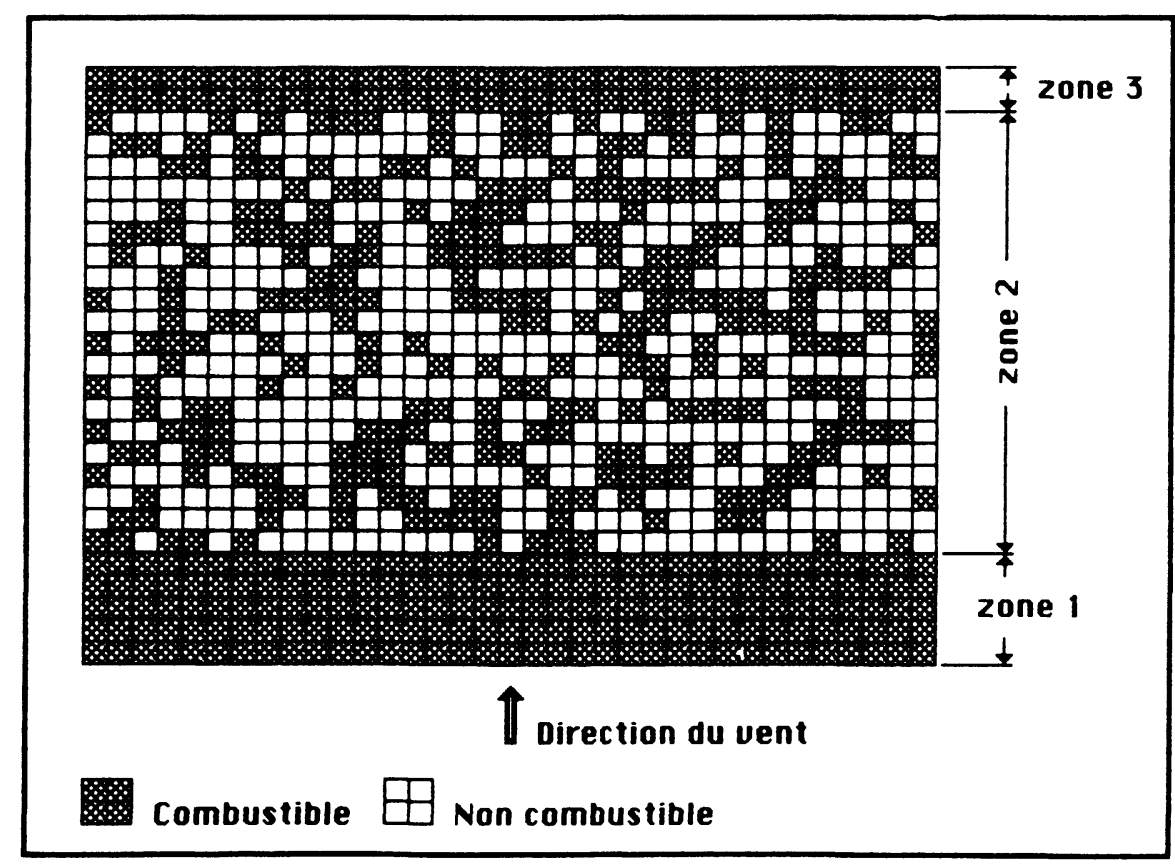

Fig. 1. 
delà du seuil de percolation, c'est-à-dire pour $q<q_{\mathrm{c}}$ ). Pour cette configuration de réseau (réseau carré de sites avec interactions possibles uniquement entre les premiers plus proches voisins), le seuil théorique de percolation est de l'ordre de $q_{\mathrm{c}}=$ 0,41 . Les dix expériences faites au-dessous de ce pourcentage confirment toutes que le feu traverse complètement la zone 2 et enflamme la zone 3 . Pour $q=0,45$, c'est-à-dire non loin du seuil théorique, le feu traverse ou non la zone 2 selon que celle-ci comporte ou pas un amas « infini », qui connecte les zones 1 et 3 (voir sur la photo 1 un cas de tirage où le feu ne traverse pas la zone 2).

Pour cette catégorie d'expériences le modèle de percolation d'invasion, introduit en premier pour décrire le drainage quasistatique d'un milieu poreux par un fluide non mouillant [12], décrit donc bien la transition propagation/non-propagation du feu à travers une zone de largeur donnée. Une expérience sur un réseau hexagonal de sites triangulaires a permis de retrouver ces mêmes résultats autour de la valeur différente du seuil de ce réseau $(q=0,35$ avec $\left.q_{\mathrm{c}}=0,30\right)$.

2) Avant d'étudier l'effet du vent, cette fois uniquement sur réseau carré, nous avons déterminé la vitesse du courant d'air à créer sur le banc expérimental, qui conduisait aux vitesses de propagation de la flamme les plus élevées sur le combustible déjà utilisé pour le réseau carré précédent. Ce courant d'air est produit par une soufflerie construite spécialement dont le jet horizontal à la sortie, est de $80 \mathrm{~cm} \times 2 \mathrm{~cm}$. La vitesse peut être considérée comme quasi-uniforme sur toute l'étendue du modèle. La mise à feu s'effectue de la même façon que pour les expériences sans vent. La soufflerie est mise en route uniquement lorsque le feu atteint la troisième ligne de la zone 1 . Nous laissons ensuite le feu se propager jusqu'à extinction.

Pour une vitesse de $0,8 \mathrm{~m} . \mathrm{s}^{-1}$ nous avons mis en évidence que, pour des pourcentages supérieurs à $q=0,65$ le feu ne traverse pas la zone 2 (voir photo 2). Pour des pourcentages inférieurs $(0,63$; $0,60 ; 0,55)$, le feu traverse toujours la zone 2 . L'effet du vent apparaît clairement sur les photos 3 et 4 , pour une même distribution à $q=0,60$. La sensibilité à la vitesse du courant d'air qui nous a conduit au choix de $0,8 \mathrm{~m} . \mathrm{s}^{-1}$, a été plus particulièrement étudiée sur une répartition à $q=0,63$. Pour des vitesses comprises entre 0,8 et $1,0 \mathrm{~m} . \mathrm{s}^{-1}$ le feu atteint la zone 3 . Pour des vitesses inférieures à $0,8 \mathrm{~m} \cdot \mathrm{s}^{-1}$ ou supérieures à $1,0 \mathrm{~m} \cdot \mathrm{s}^{-1}$ le feu s'arrête à l'intérieur de la zone 2 . Il serait intéressant de déterminer dans un travail ultérieur la variation non monotone du seuil de percolation en fonction de la vitesse du vent ainsi que le mécanisme local de propagation de la combustion par la flamme.

Les études en présence de vent appellent plusieurs remarques susceptibles de guider une analyse théori- que ultérieure. On se trouve évidemment dans un cas de transport dirigé [13]. La percolation dirigée a fait l'objet de nombreuses études et ses caractéristiques critiques, différentes de celles de la percolation usuelle, sont bien connues.

Par ailleurs la propagation ne se fait plus entre plus proches voisins mais dans certains cas entre deux sites distants de 3 mailles suggérant une correspondance avec le transport activé dans des conducteurs mentionné plus haut. Il est ainsi possible de décrire une probabilité continue de sauter d'un site à un autre fonction de la séparation, de l'orientation des sites par rapport au vent, voire d'un paramètre équivalent à la différence d'énergie entre les niveaux électroniques qui pourraient traduire des effets de combustion plus facile si la dénivelée entre le site de départ et d'arrivée est nulle ou positive.

Le problème de la propagation en combustion est donc à la fois corrélé, non local et dirigé. Le fait que nous n'avons qu'une observation de l'état final du système, ne permet pas d'isoler la contribution respective de chacun de ces caractères. Le caractère dirigé apparaît dans les régions notées $\mathrm{A}$ sur les photos 2 et 4 . Le caractère non local, correspondant à la situation de saut activé dans les semiconducteurs désordonnés, est constaté à la comparaison des photos 3 et 4 où, en présence de vent, des amas de combustible non connectés entrent quand même en combustion. Cette contagion à distance est d'autant plus facile que l'amas contagieux est grand et donc constitue un foyer important (dans les régions $B$ de la photo 4 la taille de l'amas combustible amont permet la contagion à distance alors que dans les régions $\mathrm{C}$ de la photo 2 le feu ne se propage pas du fait de la taille réduite des amas combustibles en amont). Ce résultat suggère une correspondance avec les modèles de percolation corrélée où la probabilité d'occupation d'un site est fonction de celle des sites voisins [14]. Peut-être de façon plus précise, un modèle de percolation de «bootstrap » conduisant pour une probabilité donnée à une transition brutale de percolation (et non plus continue), serait appropriée [15]. Dans ce modèle on part d'une probabilité $p(=1-q)$ de sites occupés. Ensuite tous les sites qui n'ont pas $n$ proches voisins $(n=1,2,3 \ldots)$ sont rendus inoccupés. Le processus est itéré jusqu'à ce qu'il en résulte une configuration où chaque site a $n$ voisins occupés. Il résulte de cette loi une variation du seuil par rapport à la percolation usuelle ainsi que, de façon plus importante, du comportement critique. En particulier pour $n \geqq 3$ la transition est du premier ordre en concentration. L'hystérésis résultant n'est pas sans analogue dans les problèmes de propagation des feux.

Il peut être intéressant à ce niveau de faire nettement la distinction entre les effets dus à la directivité et celui de corrélation (illustré par le modèle «bootstrap ») qui, tous deux, participent au 


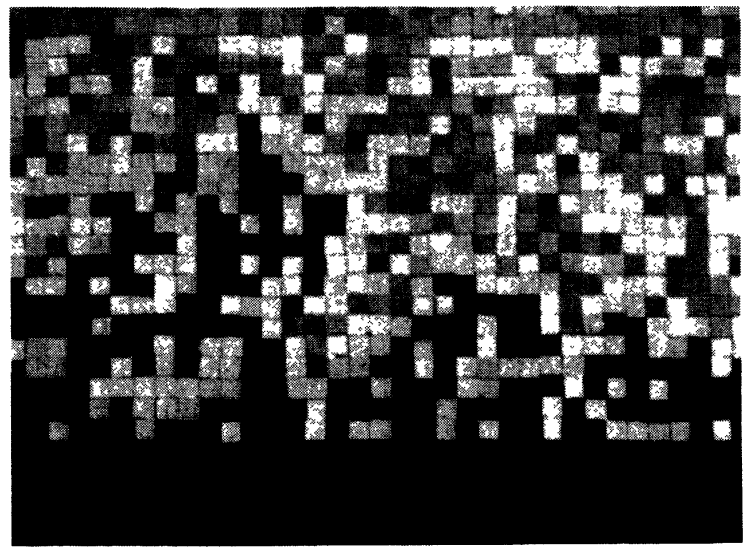

Photo 1 .

$$
q=0,45
$$$$
v=0
$$

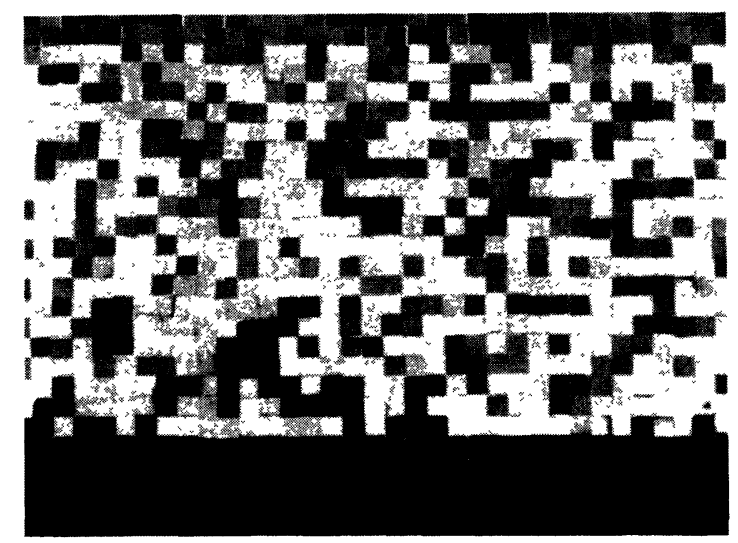

Photo 3.

$v=0$

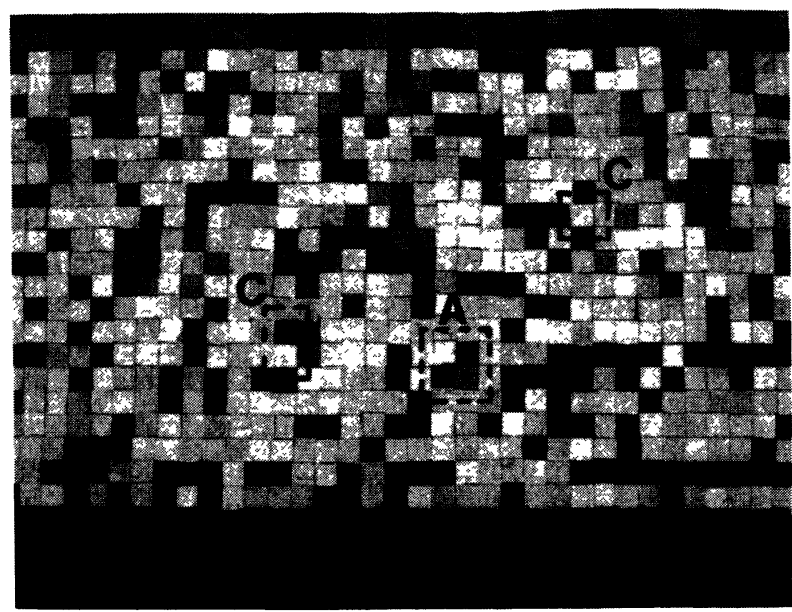

Photo 2.

$q=0,65$

$v=0,8 \mathrm{~m} \cdot \mathrm{s}^{-1}$

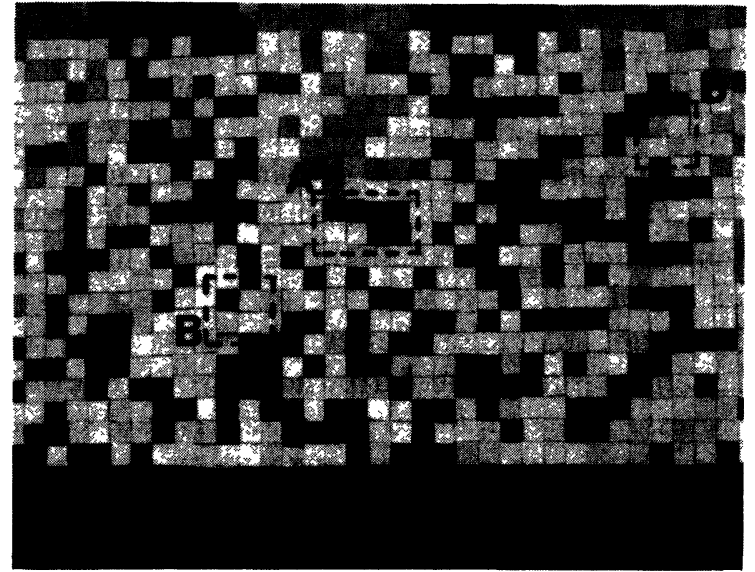

Photo 4.

$$
\begin{aligned}
& q=0,60 \\
& v=0,8 \mathrm{~m} \cdot \mathrm{s}^{-1}
\end{aligned}
$$

Photos 1-4. - $q$ est le rapport du nombre d'éléments non combustibles au nombre de sites total. Les éléments non combustibles apparaissent en plus clair sur les photos, les éléments combustibles non brûlés légèrement foncés et les éléments combustibles brûlés noirs. $v$ est la vitesse du vent (du bas vers le haut des photos). Les quatre photos représentent l'état final après combustion du modèle pour des valeurs différentes de la vitesse du vent et du pourcentage $q$. Sur toutes les photos, les éléments non combustibles recouverts de suie au terme des expériences (et donc difficiles à distinguer des éléments brûlés), ont été retournés pour des raisons de clarté. La photo 1 correspond à une «invasion » de la combustion en dessous du seuil de percolation théorique pour un réseau de sites carré avec interaction entre proches voisins. La photo 2 correspond à un pourcentage de sites non combustibles juste supérieur à la valeur de la concentration critique pour un vent de $0,8 \mathrm{~m} \cdot \mathrm{s}^{-1}$. La contagion ne se fait plus seulement entre proches voisins. Les photos 3 et 4 correspondent au même tirage avec une concentration $q=0,60$ en l'absence (3) et en présence (4) d'un vent de $0,8 \mathrm{~m} . \mathrm{s}^{-1}$. Sur les photos 2 et 4 les régions notées A montrent que la contagion est rendue difficile dans la direction perpendiculaire à celle du vent. Dans tous les cas où les zones brûlées ont des bras dirigés vers l'amont, ceux-ci ont été brâlés du bas vers le haut. Sur la photo 2 les régions notées $\mathrm{C}$ et sur la photo 4 celles notées $\mathrm{B}$ montrent les caractères non local et corrélé de la contagion. En B l'amas amont, large, permet l'allumage de l'amas aval malgré les 2 sites non combustibles d'écart, alors qu'en $\mathrm{C}$ avec un seul site d'écart mais avec un plus petit amas en amont l'allumage ne se fait pas.

[ $q$ is the fraction of non combustible elements. The non combustible elements appear in most light on the pictures, the unburnt combustible elements appear lightly dark and the burnt ones are black. $v$ is the velocity of the wind (from bottom to the top of the pictures). The four pictures represent the final state of the model after combustion, for different values of the wind velocity and fraction values $q$. On all the pictures, the non combustible elements, covered with soot after the experiments and, therefore, difficult to distinguish from the burnt elements, were turned upside-down for clarity. Picture 1 shows an «invasion » of the combustion below the theoretical site percolation threshold for a square lattice with nearest neighbour interaction. Picture 2 corresponds to a fraction $q$ just above the critical value for a wind of $0,8 \mathrm{~m} \cdot \mathrm{s}^{-1}$. The contagion does not take place only between nearest neighbours. Pictures 3 and 4 correspond to the same configuration for $q=0.6$ in the absence (3) and in the presence (4) of wind. In pictures 2 and 4 , the regions marked A show that the contagion is made difficult in the direction perpendicular to the direction of the wind. In all cases where burnt zones have upstream arms, the latter were burnt from bottom to top. In picture 2 , the regions marked $C$ and on picture 4 those marked B, show the non local and correlated characters of the contagion. In B, the upper cluster allows the lighting of the lower cluster, in spite of the non combustible 2 site gap, while in $\mathrm{C}$ with a single site gap, the lighting does not occur.] 
processus de propagation du feu, et ceux dus à des effets localement corrélés ou encore à des effets non locaux (propagation aux seconds, troisièmes, ... proches voisins). Les seconds problèmes entraînent une modification des seuils critiques mais, en aucune façon, ne changent la classe d'universalité du problème. Par contre la présence ou l'absence des premières propriétés se traduisent par des modifications des exposants critiques, éventuellement même par des changements d'ordre de transition.

Un modèle numérique prenant en compte à la fois les effets de direction, de bootstrap et la non-localité peut être aisément formulé et, à part pour les effets de non-localité, peut se prêter à des calculs d'automates cellulaires.

Notons que ces approches, ainsi que nos expériences dans lesquelles la combustion des briquettes se fait sur un temps suffisamment long, ne présentent pas de caractéristique critique dynamique. La situation serait très différente dans le cas où, par exemple, les briquettes seraient de très faible épaisseur et où il serait nécessaire de tenir compte de la simultanéité de combustion de sites dans un modèle de percolation corrélée comme dans le modèle de [9].

Ce travail fait partie d'un projet plus long de recherche appliquée (mené par J. N.) sur la propagation des incendies. Des expériences à échelle intermédiaire (lits de fibre de bois de $2 \mathrm{~m} \times 3 \mathrm{~m}$ ) ainsi qu'un programme sur site réel $\left(800 \mathrm{~m}^{2}\right)$ ont été conduites. Leur analyse et interprétation sont en cours.

\section{Remerciements.}

Ce travail a pu être réalisé grâce au soutien financier de la société Cerberus-Guinard, 78530 Buc, France. Nous voudrions remercier plus particulièrement son directeur général R. Fondraz et son directeur scientifique J. Duboys pour l'intérêt qu'ils ont porté à ces travaux.

Nous remercions également S. Roux, pour ses nombreux conseils et critiques ainsi que le professeur J. Pantaloni pour les échanges d'information sur les incendies de forêt.

\section{Bibliographie}

[1] Rothermel R. C., USDA Forest Service Res. Paper Int. 115 (1972);

Albini F. A., Forest and Range Expt. Station; U.S. dept. of Agriculture (1976). Ces deux exemples font partie d'une série de travaux faits en particulier autour du U.S. fire department et de départements analogues au Canada et en Australie. Ces modèles, construits en utilisant les éléments de la topographie locale, de la nature du sol et de la végétation, conduisent, en fonction des conditions météorologiques à un instant donné, à des prévisions de la propagation des incendies de forêt. Ils sont utilisés actuellement en situation réelle.

[2] Lee S. L. et Hellman J. M., Adv. Heat Transfer 10 (1974) 219 ;

LeE S. L. et Emmons H. W., J. Fl. Mech. 11 (1961) 353.

[3] L'exemple d'un vaste incendie autour de Calvi en juillet 1977, remplissant bien ces conditions, a fait l'objet d'une analyse non publiée (E. Guyon). A cause de la grande hétérogénéité du relief et de la végétation, la propagation du feu, qui s'est poursuivi sur plusieurs jours, a suivi un contour irrégulier avec aussi un rythme irrégulier, fonction des hétérogénéités locales. Cette situation est tout à fait à l'opposé des expériences voulues (projet Flambeau) ou subies sur le continent américain sur de vastes étendues forestières [1].
[4] GUYON E., Vie académique C.R.A.S. (1982) p. 27 ; GuYON E., jeux de réseaux (Editions du CNRS) 1986, p. 115.

[5] ShKlovski B. I. et Efros A. L., Electronic properties of doped semiconductors (Springer-Verlag) 1984, Chaps. 8 et 9.

[6] Friedman L. et Pollak M., Philos. Mag. B 38 (1978) 173.

[7] Stauffer D., Introduction to Percolation Theory (Taylor and Francis, London) 1985.

[8] Grassberger P., Math. Biosci. 63 (1983) 157.

[9] McKay G. et Jan N., J. Phys. A 17 (1984) L757.

[10] Albinet G., Searby G. et Stauffer D., J. Phys. France 47 (1986) 1.

[11] Von Niessen W. et Blumen A., J. Phys. A 19 (1986) L289.

[12] Lenormand R. et Bories S., C.R.A.S., 291B (1980) 279.

Wilkinson D. et Willemsen J., J. Phys. A 16 (1983) 3365.

[13] KinZel W., Percolation structures and processes. Eds. G. Deutscher, R. Zallen et J. Adler, Ann. Isr. Phys. Soc. 5 p. 425.

[14] Evans J. W., J. Phys. A 20 (1987) 6487.

Chalupa J., Leath P. L. et Reich G. R., J. Phys. C 12 (1979) L31.

[15] Aizemman M. et Lebowitz J. L., Math. Problems in Stat. Phys. (Edinbourg) 1987. 